[30] H. Xiong, "Geometric mechanics, ideal hydrodynamics, and the locomotion of planar shape-changing aquatic vehicles," Ph.D. dissertation, Univ. Illinois at Urbana-Champaign, 2007.

[31] C. Zhang, D. Arnold, N. Ghods, A. Siranosian, and M. Krstic, "Source seeking with nonholonomic unicycle without position measurement and with tuning of forward velocity," Syst. Control Lett., vol. 56, pp. 245-252, 2007.

[32] C. Zhang, A. Siranosian, and M. Krstic, "Extremum seeking for moderately unstable systems and for autonomous vehicle target tracking without position meas," Automatica, vol. 43, pp. 1832-1839, 2007.

[33] X. Zhang, D. Dawson, W. Dixon, and B. Xian, "Extremum seeking nonlinear controllers for a human exercise machine," in Proc. IEEE Conf. Decis. Control, 2004, pp. 3950-3955.

\section{Stabilization of a Hierarchical Formation of Unicycle Robots with Velocity and Curvature Constraints}

\author{
Luca Consolini, Member, IEEE, \\ Fabio Morbidi, Student Member, IEEE, \\ Domenico Prattichizzo, Member, IEEE, and Mario Tosques
}

\begin{abstract}
The paper proposes a new geometric approach to the stabilization of a hierarchical formation of unicycle robots. Hierarchical formations consist of elementary leader-follower units disposed on a rooted tree: each follower sees its relative leader as a fixed point in its own reference frame. Robots' linear velocity and trajectory curvature are forced to satisfy some given bounds. The major contribution of the paper is to study the effect of these bounds on the admissible trajectories of the main leader. In particular, we provide recursive formulas for the maximum velocity and curvature allowed for the main leader, so that the robots can achieve the desired formation while respecting their input constraints. An original formation control law is proposed and the asymptotic stabilization is proved. Simulation experiments illustrate the theory and show the effectiveness of the proposed designs.
\end{abstract}

Index Terms-Formation control, mobile robots, motion control, multiagent systems, nonlinear systems.

\section{INTRODUCTION}

Recent years have witnessed a growing interest in robotics, in motion coordination and cooperative control of multiagent systems [1]-[3]. In this respect, several new problems, such as, e.g., consensus [4], [5], rendezvous [6], [7], coverage [8], connectivity maintenance [9], [10], and formation control, have been formulated and solved using tools coming from computer science and control theory. Among them, for its wide range of applicability, the formation control problem received a special attention and stimulated a great deal of research [11]-[15].

Manuscript received June 4, 2009. First published September 11, 2009; current version published October 9, 2009. This paper was recommended for publication by Associate Editor W. Chung and Editor K. Lynch upon evaluation of the reviewers' comments.

L. Consolini is with the Department of Information Engineering, University of Parma, Parma 43100, Italy (e-mail: lucac@ce.unipr.it).

F. Morbidi and D. Prattichizzo are with the Department of Information Engineering, University of Siena, Siena 53100, Italy (e-mail: morbidi@dii.unisi.it; prattichizzo@dii.unisi.it).

M. Tosques is with the Department of Civil Engineering, University of Parma, Parma 43100, Italy (e-mail: mario.tosques@unipr.it).

Color versions of one or more of the figures in this paper are available online at http://ieeexplore.ieee.org.

Digital Object Identifier 10.1109/TRO.2009.2026505

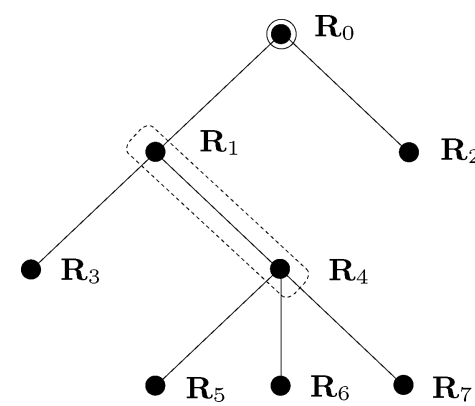

Fig. 1. Structure of a simple hierarchical formation. The main leader $\mathbf{R}_{0}$ guides the formation. One of the seven leader-follower units is highlighted: $\mathbf{R}_{1}$ is the relative leader of $\mathbf{R}_{4}$. Robots $\mathbf{R}_{5}, \mathbf{R}_{6}$, and $\mathbf{R}_{7}$ act only as followers in the formation.

By formation control, we simply mean the problem of controlling the relative position and orientation of the robots in a group while allowing the group to move as a whole. Typical working scenarios of robot formations are terrain and utilities inspection, disaster monitoring, environmental surveillance, search and rescue, structures moving and assembling, and planetary exploration. Research on formation control dealt with ground vehicles [16], [17], autonomous underwater vehicles (AUVs) [18], [19], unmanned aerial vehicles (UAVs) [20], [21], and microsatellites [22], [23].

One of the main approaches to formation control is leader-following [24]-[26]. A robot of the formation, designed as the leader, moves along a predefined trajectory, while the other robots, the followers, are to maintain a desired distance and orientation to the leader. Leaderfollower architectures are known to have poor disturbance rejection properties. In addition, the overreliance on a single agent to achieve the goal may be undesirable, especially in adverse conditions. Nevertheless, the leader-follower approach is particularly appreciated for its simplicity and scalability.

This paper extends our previous work [27] on the stabilization of a leader-follower pair of robots, to hierarchical formations of unicycles with input constraints. Hierarchical formations are characterized by elementary leader-follower units, whose interconnection is described by a rooted tree graph (see Fig. 1). The main leader $\mathbf{R}_{0}$ drives the formation, while all the other robots $\mathbf{R}_{i}$ act both as followers and leaders (with the exception of the leaves of the tree, which are only followers).

The original contribution of this paper with respect to the existing literature is twofold.

1) Each follower in the formation has to maintain a desired distance and orientation to its relative leader with respect to its own local reference frame.

2) The velocity and the curvature admissible for each follower in the formation are assumed to be bounded. The maximum velocity and curvature that are allowed for the main leader are determined through recursive formulas and a stabilizing controller is designed for each robot, so that the desired formation is asymptotically achieved and the input constraints are respected.

A peculiar feature of the proposed control strategy is that at steady state the whole formation is not rigid but changes its shape according to the motion of the main leader. Each follower's position is not fixed in the reference frame of its relative leader, but it varies in time in a suitable circle arc fixed in the relative leader's frame.

The rest of the paper is organized as follows. In Section II, some basic definitions are provided and the problem studied in the paper is formulated. In Sections III and IV, an original solution to the stabilization 


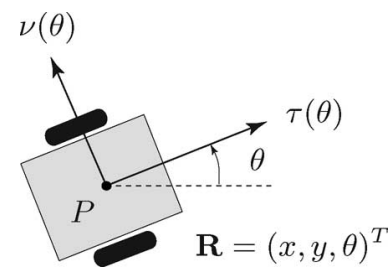

Fig. 2. Variables of the unicycle robot.

problem of a two-robot and a multirobot formation is proposed. In Section V, simulation experiments illustrate the theory and show the effectiveness of the proposed designs. In Section VI, the main contributions of the paper are summarized and future research directions are highlighted.

The following notation will be used throughout the paper:

$\forall a, b \in \mathbb{R}, \quad a \wedge b=\min \{a, b\}, \quad a \vee b=\max \{a, b\} ; \quad \forall t>0$, $\operatorname{sign}(t)=1 ; \forall t<0, \quad \operatorname{sign}(t)=-1 ; \forall x=\left(x_{1}, \ldots, x_{n}\right)^{T}, y=$ $\left(y_{1}, \ldots, y_{n}\right)^{T} \in \mathbb{R}^{n}(n \geq 1),\langle x, y\rangle=\sum_{i=1}^{n} x_{i} y_{i},\|x\|=\sqrt{\langle x, x\rangle} ;$ $\forall \theta \in \mathbb{R}, \tau(\theta)=(\cos \theta, \sin \theta)^{T}, \nu(\theta)=(-\sin \theta, \cos \theta)^{T} ; \mathcal{S}^{1}$ denotes the quotient space $\mathbb{R} / \mathscr{R}$ equipped with the canonical topology, $\mathscr{R}$ being the equivalence relation $x \sim y \Leftrightarrow x-y=2 m \pi, m \in \mathbb{Z}$.

\section{BASIC DEFINITIONS AND PROBLEM FORMULATION}

Consider the following definition of robot as a velocity-controlled unicycle model.

Definition 1: $\mathbf{R}=(x, y, \theta)^{T} \in \mathcal{C}^{1}\left([0,+\infty), \mathbb{R}^{3}\right)$ is called a unicycle robot with initial condition $\overline{\mathbf{R}} \in \mathbb{R}^{3}$ and control $(v, \omega)^{T} \in$ $\mathcal{C}^{0}\left([0,+\infty), \mathbb{R}^{2}\right)$, if the following system is verified:

$$
\left\{\begin{array}{l}
\dot{x}=v \cos \theta \\
\dot{y}=v \sin \theta \\
\dot{\theta}=\omega \\
(x(0), y(0), \theta(0))^{T}=\overline{\mathbf{R}} .
\end{array}\right.
$$

System (1) represents the differential kinematics of the unicycle robot, and $\forall t \geq 0$ such that $v(t) \neq 0, \kappa(t)=\omega(t) / v(t)$ is the curvature of the path followed by the robot at time $t$.

Let us denote the position of $\mathbf{R}$ at time $t$ by $P(t)=(x(t), y(t))^{T}$, $\theta(t)$ its heading, $\tau(\theta(t))$ the normalized velocity vector, and $\nu(\theta(t))$ the normalized vector orthogonal to $\tau(\theta(t))$. Hence, $\{\tau(\theta(t)), \nu(\theta(t))\}$ represents the robot reference frame at time $t$ (see Fig. 2).

Definition 2: The triple $(D, \Phi, \mathscr{N})$, where $D=\left(d_{1}, d_{2}, \ldots\right.$, $\left.d_{n}\right)^{T} \in \mathbb{R}^{n}, \Phi=\left(\phi_{1}, \phi_{2}, \ldots, \phi_{n}\right)^{T} \in \mathbb{R}^{n}$, and $\mathscr{N}=\left(l_{1}, l_{2}, \ldots\right.$, $\left.l_{n}\right)^{T} \in \mathbb{N}^{n}$, is said to be admissible (to make a formation), if $\forall i=$ $1, \ldots, n, d_{i}>0,\left|\phi_{i}\right|<\pi / 2$, and $l_{i} \in\{0, \ldots, i-1\}$.

The following definition introduces the notion of hierarchical formation (or $(D, \Phi, \mathscr{N})$-formation) used throughout the paper (see Fig. 3).

Definition 3: Let $(D, \Phi, \mathscr{N}) \in \mathbb{R}^{n} \times \mathbb{R}^{n} \times \mathbb{N}^{n}$ be an admissible triple. We say that $n+1$ robots $\mathbf{R}_{0}, \mathbf{R}_{1}, \ldots, \mathbf{R}_{n}$ are in $(D, \Phi, \mathscr{N})$ formation with leader $\mathbf{R}_{0}$ at time $t$, if $\forall i=1, \ldots, n$,

$$
P_{l_{i}}(t)=P_{i}(t)+d_{i} \tau\left(\theta_{i}(t)+\phi_{i}\right)
$$

and simply that $\mathbf{R}_{0}, \mathbf{R}_{1}, \ldots, \mathbf{R}_{n}$ are in $(D, \Phi, \mathscr{N})$-formation with leader $\mathbf{R}_{0}$, if (2) holds $\forall i=1, \ldots, n, \forall t \geq 0$.

$\mathbf{R}_{0}$ guides the formation and is called the main leader. $\mathbf{R}_{l_{i}}$ is the relative leader to the $i$ th follower $\mathbf{R}_{i}$, and each pair $\left(\mathbf{R}_{l_{i}}, \mathbf{R}_{i}\right)$ is referred to as a leader-follower unit. Definition 3 states that $n+1$ robots $\mathbf{R}_{0}, \mathbf{R}_{1}, \ldots, \mathbf{R}_{n}$ are in $(D, \Phi, \mathscr{N})$-formation with leader $\mathbf{R}_{0}$, if for any robot $\mathbf{R}_{i}$, the position $P_{i}$ of the follower $\mathbf{R}_{i}$ is always at distance $d_{i}$ from the position $P_{l_{i}}$ of the relative leader $\mathbf{R}_{l_{i}}$, and the



Fig. 3. Sample $(D, \Phi, \mathscr{N})$-formation with $D=\left(d_{1}, d_{2}, d_{3}\right)^{T}, \Phi=$ $\left(\phi_{1}, \phi_{2}, \phi_{3}\right)^{T}$, and $\mathscr{N}=(0,0,2)^{T}$.

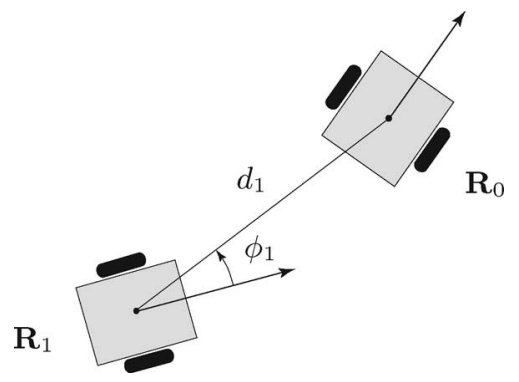

Fig. 4. $\left(d_{1}, \phi_{1}, 0\right)$-formation.

angle between vectors $\tau\left(\theta_{i}\right)$ and $P_{l_{i}}-P_{i}$ is constantly equal to $\phi_{i}$, i.e., the position $P_{l_{i}}$ of the relative leader remains fixed with respect to the reference frame $\left\{\tau\left(\theta_{i}\right), \nu\left(\theta_{i}\right)\right\}$. Notice that we use the expression hierarchical formation because giving $\mathscr{N}$ is equivalent to assign a relative leader to each robot and this defines a partial ordering in the set of the robots, which is usually called a hierarchy. Note also that usually in the literature, the follower's position is kept fixed with respect to the frame of the leader (see, e.g., [24] and [26]). On the contrary, in the proposed setup, it is the relative leader's position that is kept fixed in each follower's reference frame.

An equivalent way to state Definition 3 is the following. Set the error vector

$$
E_{i}(t)=P_{l_{i}}(t)-\left(P_{i}(t)+d_{i} \tau\left(\theta_{i}(t)+\phi_{i}\right)\right)
$$

then $\mathbf{R}_{0}, \mathbf{R}_{1}, \ldots, \mathbf{R}_{n}$ are in $(D, \Phi, \mathscr{N})$-formation with leader $\mathbf{R}_{0}$ if and only if $E_{i}(t)=0 \forall i=1, \ldots, n, \forall t \geq 0$.

It is worth noting that hierarchical formations represent rooted trees in the context of graph theory. In the special case that $l_{i}=0$ for all $i$, all the robots have $\mathbf{R}_{0}$ as relative leader, while if $l_{i}=i-1$ for all $i$, a "convoy-like" formation is obtained, i.e., a formation where the $i$ th robot has the $(i-1)$ th as relative leader. Finally, note that if $n=1$, then $D=d_{1}, \Phi=\phi_{1}, \mathscr{N}=0$, i.e., $\mathbf{R}_{0}$ and $\mathbf{R}_{1}$ are in $\left(d_{1}, \phi_{1}, 0\right)$ formation with leader $\mathbf{R}_{0}$, which is the case considered in [27] (see Fig. 4).

Definition 4: Let $(D, \Phi, \mathscr{N}) \in \mathbb{R}^{n} \times \mathbb{R}^{n} \times \mathbb{N}^{n}$ be an admissible triple. We say that $n+1$ robots $\mathbf{R}_{0}, \mathbf{R}_{1}, \ldots, \mathbf{R}_{n}$ are asymptotically in $(D, \Phi, \mathscr{N})$-formation with leader $\mathbf{R}_{0}$, if $\forall i=1, \ldots, n$,

$$
\lim _{t \rightarrow \infty} E_{i}(t)=P_{l_{i}}(t)-\left(P_{i}(t)+d_{i} \tau\left(\theta_{i}(t)+\phi_{i}\right)\right)=0 .
$$

The following problem will be studied in the next sections. 
Problem 1: Let $(D, \Phi, \mathscr{N}) \in \mathbb{R}^{n} \times \mathbb{R}^{n} \times \mathbb{N}^{n}$ be an admissible triple. Let $\mathbf{R}_{0}, \mathbf{R}_{1}, \ldots, \mathbf{R}_{n}$ be $n+1$ robots and $V_{i}, K_{i}^{-}, K_{i}^{+}$, $i=0, \ldots, n$, be real constants. Find the velocity and curvature bounds $V_{0}, K_{0}^{-}, K_{0}^{+}$of robot $\mathbf{R}_{0}$ that guarantee the existence of controls $v_{i}, \omega_{i}, i=1, \ldots, n$, for robot $\mathbf{R}_{i}$, such that for any initial condition $\overline{\mathbf{R}}_{i}$, the robots $\mathbf{R}_{0}, \mathbf{R}_{1}, \ldots, \mathbf{R}_{n}$ are asymptotically in $(D, \Phi, \mathscr{N})$ formation with leader $\mathbf{R}_{0}$ and the following constraints are satisfied: $\forall i=1, \ldots, n$, and $\forall t \geq 0$,

$$
0<v_{i}(t) \leq V_{i}, \quad K_{i}^{-} \leq \kappa_{i}(t) \leq K_{i}^{+}
$$

Roughly speaking, given the curvature and velocity bounds of each follower robot, we aim to find the bounds on the velocity and curvature of the main leader such that there exist controls for the followers, for which the robots asymptotically achieve the desired formation while respecting their own velocity and curvature constraints.

\section{Stabilization of A Two-Robot Formation}

Before dealing with Problem 1, it is worth studying a simpler case, namely the asymptotic stabilization of a two-robot formation. To understand the choice of the controls we are going to use, consider the following remark.

Remark 1: Suppose that two robots $\mathbf{R}_{0}$ and $\mathbf{R}_{1}$ are in $\left(d_{1}, \phi_{1}, 0\right)$ formation with leader $\mathbf{R}_{0}$. Then, it must be

$$
v_{1}(t)=v_{0}(t) \frac{\cos \left(\beta_{1}(t)-\phi_{1}\right)}{\cos \phi_{1}}, \quad \omega_{1}(t)=v_{0}(t) \frac{\sin \beta_{1}(t)}{d_{1} \cos \phi_{1}}
$$

where $\beta_{1}(t) \triangleq \theta_{0}(t)-\theta_{1}(t)$ is the relative heading of the two robots. In fact, since $E_{1}(t)=P_{0}(t)-\left(P_{1}(t)+d_{1} \tau\left(\theta_{1}(t)+\phi_{1}\right)\right)=0$, differentiating it, we get that $\dot{P}_{0}(t)=\dot{P}_{1}(t)+d_{1} \dot{\theta}_{1}(t) \nu\left(\theta_{1}(t)+\right.$ $\left.\phi_{1}\right)$, i.e., $v_{0}(t) \tau\left(\theta_{0}(t)\right)=v_{1}(t) \tau\left(\theta_{1}(t)\right)+d_{1} \omega_{1}(t) \nu\left(\theta_{1}(t)+\phi_{1}\right)$, which implies, multiplying by the rotation matrix $\mathcal{R}\left(-\theta_{1}(t)\right)$ $\triangleq\left(\tau\left(-\theta_{1}(t)\right), \nu\left(-\theta_{1}(t)\right)\right), \quad$ that $\quad v_{0}(t) \tau\left(\beta_{1}(t)\right)=v_{1}(t)(1,0)^{T}+$ $d_{1} \omega_{1}(t) \nu\left(\phi_{1}\right)$. Therefore, it has to be

$$
\begin{aligned}
& v_{1}=v_{0}\left[\cos \beta_{1}+\frac{\sin \beta_{1} \sin \phi_{1}}{\cos \phi_{1}}\right]=v_{0} \frac{\cos \left(\beta_{1}-\phi_{1}\right)}{\cos \phi_{1}} \\
& \omega_{1}=v_{0} \frac{\sin \beta_{1}}{d_{1} \cos \phi_{1}} .
\end{aligned}
$$

The stabilization control strategy that we are going to present consists of two steps. In the first step, $\mathbf{R}_{1}$ moves with maximum linear and angular velocities until its direction is sufficiently close to that of $\mathbf{R}_{0}$. In the second step, $\mathbf{R}_{1}$ performs the control defined in Remark 1 with an added stabilizing term in order to reduce the error asymptotically to zero. Note that since $\forall a, a_{1}, a_{2} \in \mathbb{R}^{2}$ such that $\left\langle a_{1}, a_{2}^{\perp}\right\rangle \neq 0$, $a=\left(\left\langle a, a_{2}^{\perp}\right\rangle /\left\langle a_{1}, a_{2}^{\perp}\right\rangle\right) a_{1}+\left(\left\langle a, a_{1}^{\perp}\right\rangle /\left\langle a_{2}, a_{1}^{\perp}\right\rangle\right) a_{2},\left(a_{1}^{\perp}\right.$ and $a_{2}^{\perp}$ are vectors orthogonal to $a_{1}$ and $a_{2}$, respectively), then the error vector $E_{1}$ can be decomposed with respect to vectors $\tau\left(\theta_{1}\right)$ and $\nu\left(\theta_{1}+\phi_{1}\right)$ as follows:

$$
\begin{aligned}
E_{1} & =\frac{\left\langle E_{1}, \nu\left(\theta_{1}+\phi_{1}\right)^{\perp}\right\rangle}{\left\langle\tau\left(\theta_{1}\right), \nu\left(\theta_{1}+\phi_{1}\right)^{\perp}\right\rangle} \tau\left(\theta_{1}\right)+\frac{\left\langle E_{1}, \tau\left(\theta_{1}\right)^{\perp}\right\rangle}{\left\langle\nu\left(\theta_{1}+\phi_{1}\right), \tau\left(\theta_{1}\right)^{\perp}\right\rangle} \nu\left(\theta_{1}+\phi_{1}\right) \\
& =E_{1 \tau} \tau\left(\theta_{1}\right)+E_{1 \nu} \nu\left(\theta_{1}+\phi_{1}\right)
\end{aligned}
$$

where $E_{1 \tau}=\left\langle E_{1}, \tau\left(\theta_{1}+\phi_{1}\right)\right\rangle / \cos \phi_{1}, E_{1 \nu}=\left\langle E_{1}, \nu\left(\theta_{1}\right)\right\rangle / \cos \phi_{1}$ (see Fig. 5).

Let $\Gamma_{\epsilon}^{1}=\left\{\gamma \in \mathcal{S}^{1} \mid\left(K_{0}^{-}-\epsilon\right) d_{1} \cos \phi_{1} \leq \sin \gamma \leq\left(K_{0}^{+}+\epsilon\right) d_{1}\right.$ $\left.\cos \phi_{1}\right\}$. The stabilizing controller that will be referred to in

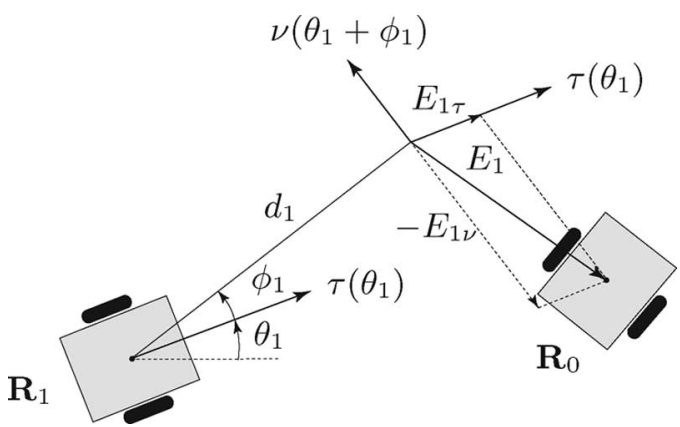

Fig. 5. Error decomposition, where $E_{1 \tau}=\left\langle E_{1}, \tau\left(\theta_{1}+\phi_{1}\right)\right\rangle / \cos \phi_{1}$ and $E_{1 \nu}=\left\langle E_{1}, \nu\left(\theta_{1}\right)\right\rangle / \cos \phi_{1}$.

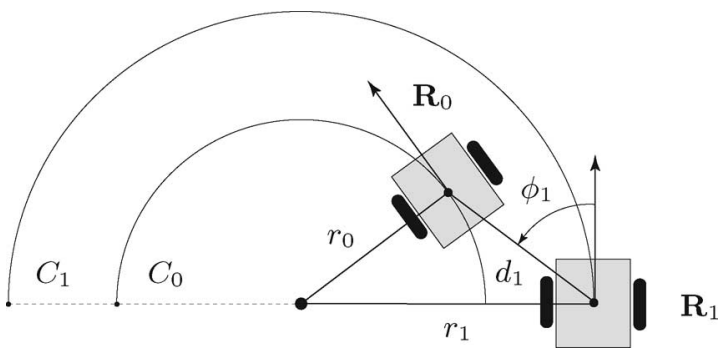

Fig. 6. $\quad \mathbf{R}_{0}$ and $\mathbf{R}_{1}$ in $=\left(d_{1}, \phi_{1}, 0\right)$-formation on two circles of radius $r_{0}$ and $r_{1}$.

Proposition 1 is given by

$v_{1}(t)= \begin{cases}V_{1}, & \text { if } \beta_{1}(t) \notin \Gamma_{\epsilon}^{1} \\ \frac{v_{0}(t) \cos \left(\beta_{1}(t)-\phi_{1}\right)}{\cos \phi_{1}}+\eta_{1}(t) E_{1 \tau}(t), & \text { if } \beta_{1}(t) \in \Gamma_{\epsilon}^{1}\end{cases}$

$\omega_{1}(t)= \begin{cases}V_{1} K_{1}^{+}, & \text {if } \beta_{1}(t) \notin \Gamma_{\epsilon}^{1} \text { and } K_{1}^{+} \geq 0 \\ V_{1} K_{1}^{-}, & \text {if } \beta_{1}(t) \notin \Gamma_{\epsilon}^{1} \text { and } K_{1}^{+}<0 \\ \frac{v_{0}(t) \sin \beta_{1}(t)}{d \cos \phi_{1}}+\eta_{1}(t) \frac{E_{1 \nu}(t)}{d_{1}}, & \text { if } \beta_{1}(t) \in \Gamma_{\epsilon}^{1}\end{cases}$

which adds correcting terms proportional to the error components to the controls $v_{1}, \omega_{1}$ in (3) [see the proof of Proposition 1 in the Appendix for the definition of the proportionality factor $\left.\eta_{1}(t)\right]$. Note that by decomposing the error $E_{1}$ into the components $E_{1 \tau}$ and $E_{1 \nu}$, we decoupled the stabilizing control of robot $\mathbf{R}_{1}$. In fact, in (5) $v_{1}$ only depends on $E_{1 \nu}$ and in (6) $\omega_{1}$ only depends on $E_{1 \tau}$. In the Appendix, we will prove that $\Gamma_{\epsilon}^{1}$ has the following property: if $\beta_{1}(\bar{t}) \in \Gamma_{\epsilon}^{1}$, then, $\beta_{1}(t) \in \Gamma_{\epsilon}^{1} \forall t \geq \bar{t}$, i.e., $\Gamma_{\epsilon}^{1}$ is a positive controlled invariant set for $\beta_{1}$. The first step of the stabilization strategy ensures that there exists a time $\bar{t}$ such that $\beta_{1}(\bar{t}) \in \Gamma_{\epsilon}^{1}$. In this way, at time $\bar{t}$ the heading of $\mathbf{R}_{1}$ is sufficiently close to the heading of $\mathbf{R}_{0}$. The second step of the control strategy guarantees that $\beta_{1}$ remains in $\Gamma_{\epsilon}^{1}$ and that the two robots asymptotically converge to the desired formation.

To understand the genesis of some of the constants and constraints that appear in the statement of Proposition 1, consider the situation presented in Fig. 6, where the robots $\mathbf{R}_{0}$ and $\mathbf{R}_{1}$ are in $\left(d_{1}, \phi_{1}, 0\right)$ formation and follow two circles $C_{0}$ and $C_{1}$ of radius $r_{0}, r_{1}$ and curvature $K_{0}>0, K_{1}>0$, respectively. If $d_{1}>0,\left|\phi_{1}\right|<\pi / 2$ and 
$K_{0}<1 /\left(d_{1}, \cos \phi_{1}\right)$, the following relations hold true:

$$
\begin{aligned}
r_{1} & =d_{1} \sin \phi_{1}+\sqrt{r_{0}^{2}-d_{1}^{2} \cos ^{2} \phi_{1}} \\
K_{1} & =\frac{1}{d_{1} \sin \phi_{1}+\sqrt{\left(1 / K_{0}^{2}\right)-d_{1}^{2} \cos ^{2} \phi_{1}}} .
\end{aligned}
$$

Indeed, as shown in Proposition 1, the value of $K_{1}$ given in (7) is precisely the maximum positive curvature of a follower robot whose leader is following a path of maximum positive curvature less than $K_{0}$. An analogous reasoning can be made for negative curvatures. The following definition is introduced to provide concise statements in Proposition 1.

Definition 5: Let $d>0$ and $\phi:|\phi|<\pi / 2$. Define the following strictly increasing monotone functions: $\forall, \kappa \in \mathbf{R}$ such that $|\kappa| \leq$ $1 /(d \cos \phi)$, set,

$$
\chi_{d, \phi}(\kappa)=\frac{1}{d \sin \phi+(\operatorname{sign} \kappa) \sqrt{\left(1 / \kappa^{2}\right)-d^{2} \cos ^{2} \phi}}
$$

and $\forall \kappa \in \mathbb{R}$, set,

$$
\chi_{d, \phi}^{-1}(\kappa)=\frac{\operatorname{sign} \kappa}{\sqrt{d^{2} \cos ^{2} \phi+((1 / \kappa)-d \sin \phi)^{2}}}
$$

with the convention that $\chi_{d, \phi}(0)=\chi_{d, \phi}^{-1}(0)=0$.

Therefore, in the example of Fig. $6, K_{1}=\chi_{d_{1}, \phi_{1}}\left(K_{0}\right)$ [see (7)] and $K_{0}=\chi_{d_{1}, \phi_{1}}^{-1}\left(K_{1}\right)$.

Let $d_{1}>0, \phi_{1}:\left|\phi_{1}\right|<\pi / 2$, and let $W_{0}, V_{0}, K_{0}^{-}, K_{0}^{+}, V_{1}, K_{1}^{-}$, $K_{1}^{+}$be suitable constants such that

$0<W_{0}, V_{0}, V_{1}, K_{1}^{-} \leq K_{1}^{+}$

$\left.\begin{array}{l}\text { if } \phi_{1}<0,-\frac{1}{d_{1} \cos \phi_{1}} \\ \text { if } \phi_{1} \geq 0,-\frac{1}{d_{1}}\end{array}\right\}<K_{0}^{-} \leq K_{0}^{+}< \begin{cases}\frac{1}{d_{1} \cos \phi_{1}}, & \text { if } \phi_{1}>0 \\ \frac{1}{d_{1}}, & \text { if } \phi_{1} \leq 0 .\end{cases}$

Set $\breve{K}_{0}^{ \pm}=\chi_{d_{1}, \phi_{1}}^{-1}\left(K_{1}^{ \pm}\right)$and

$$
\begin{aligned}
\breve{V}_{0} & =V_{1} \cos \phi_{1}\left(\operatorname { c o s } \left(0 \wedge\left(\arcsin \left(K_{0}^{+} d_{1} \cos \phi_{1}\right)-\phi_{1}\right)\right.\right. \\
& \left.\left.\wedge\left(\phi_{1}-\arcsin \left(K_{0}^{-} d_{1} \cos \phi_{1}\right)\right)\right)\right)^{-1} .
\end{aligned}
$$

The proof of the next proposition is given in the Appendix.

Proposition 1: In the previous notation and hypotheses, let $\mathbf{R}_{0}$ be a robot that satisfies the following constraints for all $t \geq 0$ :

$$
0<W_{0} \leq v_{0}(t) \leq V_{0}, \quad K_{0}^{-} \leq \kappa_{0}(t) \leq K_{0}^{+} .
$$

If the following conditions are satisfied:

$$
\breve{K}_{0}^{-}<K_{0}^{-} \leq K_{0}^{+}<\breve{K}_{0}^{+}, \quad V_{0}<\breve{V}_{0}
$$

then for any initial condition $\overline{\mathbf{R}}_{1}$ of the robot $\mathbf{R}_{1}$, the controls $v_{1}, \omega_{1}$ given by (5) and (6) are such that

$$
\lim _{t \rightarrow \infty} E_{1}(t)=0
$$

and the following constraints are verified for robot $\mathbf{R}_{1}$ :

$$
0<W_{1} \leq v_{1}(t) \leq V_{1}, \quad K_{1}^{-} \leq \kappa_{1}(t) \leq K_{1}^{+} \quad \forall t \geq 0
$$

where

$$
\begin{aligned}
W_{1}= & \frac{W_{0}}{2 \cos \phi_{1}} \cos \left(\left(\arcsin \left(\left(K_{0}^{+}+\epsilon\right) d_{1} \cos \phi_{1}\right)-\phi_{1}\right)\right. \\
& \left.\vee\left(\phi_{1}-\arcsin \left(\left(K_{0}^{-}-\epsilon\right) d_{1} \cos \phi_{1}\right)\right)\right)
\end{aligned}
$$

and $\epsilon>0$ is a sufficiently small constant. Furthermore, $\forall \epsilon>0$, $\exists \bar{t} \geq 0: \forall t \geq \bar{t}$

$$
\begin{gathered}
\arcsin \left(\left(K_{0}^{-}-\epsilon\right) d_{1} \cos \phi_{1}\right) \leq \theta_{0}(t)-\theta_{1}(t) \\
\leq \arcsin \left(\left(K_{0}^{+}+\epsilon\right) d_{1} \cos \phi_{1}\right) .
\end{gathered}
$$

Proposition 1 states that given a follower robot $\mathbf{R}_{1}$ with velocity and curvature constrained by (12), if the leader $\mathbf{R}_{0}$ maneuvers with velocity and curvature sufficiently bounded, then the control law given by (5) and (6) allows $\mathbf{R}_{1}$ to asymptotically reach the formation for every initial state. The bounds on the leader's velocity and curvature [see conditions (8) and (10)] depend on the formation's parameters $d_{1}, \phi_{1}$, and on the follower's capability of maneuver.

Note that condition (14) states that the difference $\theta_{0}(t)-\theta_{1}(t)$ between the robots' headings remains bounded during the motion. The following remark gives a geometric interpretation to this property.

Remark 2: Let

$$
\mathcal{A}_{d}\left(\delta, \sigma_{1}, \sigma_{2}\right)=\left\{d \tau(\theta) \mid \delta+\sigma_{1} \leq \theta \leq \delta+\sigma_{2}\right\}
$$

be the arc of circle centered in the origin, radius $d$, angle of the reference axis $\delta$, aperture $\sigma_{2}-\sigma_{1}$, and let $\mathcal{A}_{d}^{\epsilon}\left(\delta, \sigma_{1}, \sigma_{2}\right)=\left\{z \in \mathbb{R}^{2} \mid \exists z^{\prime} \in\right.$ $\left.\mathcal{A}_{d}\left(\delta, \sigma_{1}, \sigma_{2}\right):\left\|z^{\prime}-z\right\|<\epsilon\right\}$ be the $\epsilon$-neighborhood of $\mathcal{A}_{d}\left(\delta, \sigma_{1}, \sigma_{2}\right)$. Since, $\forall t \geq 0$,

$$
\begin{aligned}
P_{1}(t) & =P_{0}(t)-d_{1} \tau\left(\theta_{1}(t)+\phi_{1}\right)-E_{1}(t) \\
& =P_{0}(t)+d_{1} \tau\left(\theta_{1}(t)+\phi_{1}+\pi\right)-E_{1}(t) \\
& =P_{0}(t)+d_{1} \tau\left(\left(\theta_{0}(t)+\phi_{1}+\pi\right)-\left(\theta_{1}(t)-\theta_{0}(t)\right)\right)-E_{1}(t)
\end{aligned}
$$

we immediately deduce from (11) and (14) that the following property holds true:

$$
\begin{aligned}
\forall \epsilon> & 0, \exists \bar{t}: \forall t \geq \bar{t}, \quad P_{1}(t) \in P_{0}(t)+\mathcal{A}_{d_{1}}^{\epsilon}\left(\theta_{0}(t)+\phi_{1}+\pi,\right. \\
& \left.-\arcsin \left(K_{0}^{+} d_{1} \cos \phi_{1}\right),-\arcsin \left(K_{0}^{-} d_{1} \cos \phi_{1}\right)\right) .
\end{aligned}
$$

In other words, even if $P_{1}$ is not, in general, fixed in the leader's reference frame $\left\{\tau\left(\theta_{0}(t)\right), \nu\left(\theta_{0}(t)\right)\right\}$ during its motion, given any $\epsilon>0, P_{1}(t)$ belongs eventually to the $\epsilon$-neighborhood of the arc of circle $\mathcal{A}_{d_{1}}\left(\theta_{0}(t)+\phi_{1}+\right.$ $\left.\pi,-\arcsin \left(K_{0}^{+} d_{1} \cos \phi_{1}\right),-\arcsin \left(K_{0}^{-} d_{1} \cos \phi_{1}\right)\right)$, which is fixed with respect to the reference frame of robot $\mathbf{R}_{0}$ (see Fig. 7, where $\sigma_{1}=-\arcsin \left(K_{0}^{+} d_{1} \cos \phi_{1}\right), \sigma_{2}=-\arcsin \left(K_{0}^{-} d_{1} \cos \phi_{1}\right)$, and $\delta=\theta_{0}(t)+\phi_{1}+\pi$ in the case of $\left.K_{0}^{-}<0<K_{0}^{+}\right)$.

Remark 3: In [27, Th. 1], it has been proved that conditions (8) and (10) with the weak inequalities are necessary and sufficient for the existence of controls that satisfy the constraints and maintain the vehicles exactly in $\left(d_{1}, \phi_{1}, 0\right)$-formation, i.e., $E_{1}(t)=0 \forall t \geq 0$.

\section{Stabilization of A Multirobot Formation}

In this section, we extend the results presented in Section III to multirobot formations. The following definitions are introduced to provide concise statements in Theorem 1.

Definition 6: Let $(D, \Phi, \mathscr{N}) \in \mathbb{R}^{n} \times \mathbb{R}^{n} \times \mathbb{N}^{n}$ be an admissible triple. For every $i=0, \ldots, n$, let

$$
\mathscr{L}_{i}=\left\{j \mid i<j \leq n \quad \text { and } \quad l_{j}=i\right\}
$$




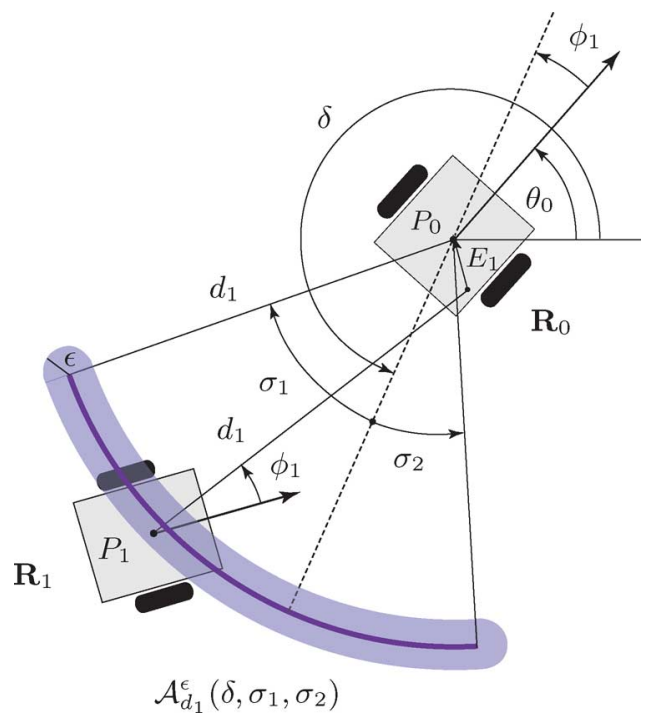

Fig. 7. $\epsilon$-Neighborhood of the arc of circle $\mathcal{A}_{d_{1}}\left(\delta, \sigma_{1}, \sigma_{2}\right)$.

be the set of the indexes $j$ such that $\mathbf{R}_{i}$ is the relative leader of $\mathbf{R}_{j}$. Recall that $\forall i=1, \ldots, n, l_{i}$ denotes the index of the robot that is the leader of $\mathbf{R}_{i}$

Note that $\mathbf{R}_{i}$ is a relative leader if and only if $\mathscr{L}_{i} \neq \emptyset$, and it always holds that $\mathscr{L}_{0} \neq \emptyset$ and $\mathscr{L}_{n}=\emptyset$.

Definition 7: Let $K_{i}^{-}, K_{i}^{+}, i=1, \ldots, n$, be $2 n$ real constants. Set $\forall i=n, n-1, \ldots, 1$,

$$
\begin{aligned}
\breve{K}_{i}^{-} & =\max \left\{K_{i}^{-}, \chi_{d_{j}, \phi_{j}}^{-1}\left(\breve{K}_{j}^{-}\right) \mid j \in \mathscr{L}_{i}\right\} \\
\breve{K}_{i}^{+} & =\min \left\{K_{i}^{+}, \chi_{d_{j}, \phi_{j}}^{-1}\left(\breve{K}_{j}^{+}\right) \mid j \in \mathscr{L}_{i}\right\}
\end{aligned}
$$

and

$$
\begin{aligned}
& \breve{K}_{0}^{-}=\max \left\{\chi_{d_{j}, \phi_{j}}^{-1}\left(\breve{K}_{j}^{-}\right) \mid j \in \mathscr{L}_{0}\right\} \\
& \breve{K}_{0}^{+}=\min \left\{\chi_{d_{j}, \phi_{j}}^{-1}\left(\breve{K}_{j}^{+}\right) \mid j \in \mathscr{L}_{0}\right\} .
\end{aligned}
$$

Note that $\breve{K}_{i}^{ \pm}=K_{i}^{ \pm}$if $\mathscr{L}_{i}=\emptyset$, and therefore, $\breve{K}_{n}^{ \pm}=K_{n}^{ \pm}$. Moreover, $\breve{K}_{i}^{-} \leq \breve{K}_{i}^{+}$if $K_{i}^{-} \leq K_{i}^{+}$, by the monotonicity of $\chi^{-1}$.

Definition 8: Let $(D, \Phi, \mathscr{N}) \in \mathbb{R}^{n} \times \mathbb{R}^{n} \times \mathbb{N}^{n}$ be an admissible triple. Suppose that $\forall i=1, \ldots, n-1$,

$$
\left.\begin{array}{l}
\text { if } \phi_{i}<0,-\frac{1}{d_{i} \cos \phi_{i}} \\
\text { if } \phi_{i} \geq 0,-\frac{1}{d_{i}}
\end{array}\right\}<K_{l_{i}}^{-} \leq K_{l_{i}}^{+}< \begin{cases}\frac{1}{d_{i} \cos \phi_{i}}, & \text { if } \phi_{i}>0 \\
\frac{1}{d_{i}}, & \text { if } \phi_{i} \leq 0\end{cases}
$$

is satisfied. Then, $\forall i=0, \ldots, n$, we have

$$
-\frac{\pi}{2}<\arcsin \left(K_{l_{i}}^{-} d_{i} \cos \phi_{i}\right)-\phi_{i}<\arcsin \left(K_{l_{i}}^{+} d_{i} \cos \phi_{i}\right)-\phi_{i}<\frac{\pi}{2} \text {. }
$$

Therefore, the following constants are well defined $\forall i=$ $n, n-1, \ldots, 1$ :

$$
\begin{gathered}
\breve{V}_{i}=\min \left\{V_{i}, \breve{V}_{j} \cos \phi_{j}\left(\operatorname { c o s } \left(0 \wedge\left(\arcsin \left(K_{i}^{+} d_{j} \cos \phi_{j}\right)-\phi_{j}\right)\right.\right.\right. \\
\left.\left.\left.\wedge\left(\phi_{j}-\arcsin \left(K_{i}^{-} d_{j} \cos \phi_{j}\right)\right)\right)\right)^{-1} \mid j \in \mathscr{L}_{i}\right\}
\end{gathered}
$$

and

$$
\begin{array}{r}
\breve{V}_{0}=\min \left\{\breve { V } _ { j } \operatorname { c o s } \phi _ { j } \left(\operatorname { c o s } \left(0 \wedge\left(\arcsin \left(K_{0}^{+} d_{j} \cos \phi_{j}\right)-\phi_{j}\right)\right.\right.\right. \\
\left.\left.\left.\wedge\left(\phi_{j}-\arcsin \left(K_{0}^{-} d_{j} \cos \phi_{j}\right)\right)\right)\right)^{-1} \mid j \in \mathscr{L}_{0}\right\} .
\end{array}
$$

Note that $\breve{V}_{i}=V_{i}$ if $\mathscr{L}_{i}=\emptyset$; therefore, $\breve{V}_{n}=V_{n}$.

In the following, we will refer to the expressions in Definitions 7 and 8 as constraints propagation formulas.

We are now ready to deal with Problem 1 and state the main theorem of the paper. The proof is reported in the Appendix. Given an admissible triple $(D, \Phi, \mathscr{N})$ and $n+1$ robots $\mathbf{R}_{0}, \mathbf{R}_{1}, \ldots, \mathbf{R}_{n}$ with preassigned velocity and curvature constraints, the following theorem presents a method to determine the corresponding bounds on the velocity and curvature of the main leader $\mathbf{R}_{0}$, such that the robots asymptotically achieve the desired formation while respecting their constraints at all times.

Theorem 1 (Main theorem): Let $(D, \Phi, \mathscr{N}) \in \mathbb{R}^{n} \times \mathbb{R}^{n} \times \mathbb{N}^{n}$ be an admissible triple, $\mathbf{R}_{0}, \mathbf{R}_{1}, \ldots, \mathbf{R}_{n}$ be $n+1$ robots, and $V_{i}>0$, $K_{i}^{-} \leq K_{i}^{+}, i=0, \ldots, n$, be $3(n+1)$ real constants, such that $\forall i=$ $1, \ldots, n$, condition (15) is satisfied. Suppose that there exists a constant $W_{0}$ such that

$$
0<W_{0} \leq v_{0}(t) \leq V_{0}, \quad K_{0}^{-} \leq \kappa_{0}(t) \leq K_{0}^{+} .
$$

Let $\breve{K}_{0}^{-}, \breve{K}_{0}^{+}$, and $\breve{V}_{0}$ be as given in Definitions 7 and 8 . If the following conditions are satisfied:

$$
\breve{K}_{0}^{-}<K_{0}^{-} \leq K_{0}^{+}<\breve{K}_{0}^{+}, \quad V_{0}<\breve{V}_{0}
$$

then there exists $2 n$ suitable controls $v_{i}, \omega_{i}, i=1, \ldots, n$, such that the robots $\mathbf{R}_{0}, \mathbf{R}_{1}, \ldots, \mathbf{R}_{n}$ are asymptotically in $(D, \Phi, \mathscr{N})$-formation with leader $\mathbf{R}_{0}$, and the following conditions are fulfilled:

$$
0<W_{i} \leq v_{i}(t) \leq V_{i}, \quad K_{i}^{-} \leq \kappa_{i}(t) \leq K_{i}^{+} \quad \forall t \geq 0
$$

where $W_{i}$ is given in (29) (see the Appendix). Moreover, $\forall \epsilon>0, \exists \bar{t}$ : $\forall t \geq \bar{t}$

$$
\begin{aligned}
& P_{i}(t) \in P_{l_{i}}(t)+\mathcal{A}_{d_{i}}^{\epsilon}\left(\theta_{l_{i}}(t)+\phi_{i}+\pi,-\arcsin \left(K_{l_{i}}^{+} d_{i} \cos \phi_{i}\right)\right. \\
& \left.-\arcsin \left(K_{l_{i}}^{-} d_{i} \cos \phi_{i}\right)\right) \quad \forall i=1, \ldots, n .
\end{aligned}
$$

Remark 4: Hypothesis (17) guarantees that the leader's allowed maximum and minimum curvatures $K_{0}^{+}, K_{0}^{-}$are strictly contained in the set $\left[\breve{K}_{0}^{-}, \breve{K}_{0}^{+}\right]$of leader's curvature values that are compatible with all the followers' bounds and, similarly, that the leader's maximum velocity $V_{0}$ is lower than the maximum velocity $\breve{V}_{0}$ compatible with all the followers' constraints.

Remark 5: As shown in the proof in the Appendix, under the hypotheses of Theorem 1, if we set $\Gamma_{\epsilon}^{i}=\left\{\gamma \in \mathcal{S}^{1} \mid\left(K_{l_{i}}^{-}-\epsilon\right) d_{i} \cos \phi_{i} \leq\right.$ $\left.\sin \gamma \leq\left(K_{l_{i}}^{+}+\epsilon\right) d_{i} \cos \phi_{i}\right\}$ and $\beta_{i}(t)=\theta_{l_{i}}(t)-\theta_{i}(t)$, the controls

$$
\begin{array}{r}
v_{i}(t)= \begin{cases}V_{i}, & \text { if } \beta_{i}(t) \notin \Gamma_{\epsilon}^{i} \\
\frac{v_{l_{i}}(t) \cos \left(\beta_{i}(t)-\phi_{i}\right)}{\cos \phi_{i}}+\eta_{i}(t) E_{i \tau}(t), & \text { if } \beta_{i}(t) \in \Gamma_{\epsilon}^{i}\end{cases} \\
\omega_{i}(t)= \begin{cases}V_{i} K_{i}^{+}, & \text {if } \beta_{i}(t) \notin \Gamma_{\epsilon}^{i} \text { and } K_{i}^{+} \geq 0 \\
V_{i} K_{i}^{-}, & \text {if } \beta_{i}(t) \notin \Gamma_{\epsilon}^{i} \text { and } K_{i}^{+}<0 \\
\frac{v_{l_{i}}(t) \sin \beta_{i}(t)}{d_{i} \cos \phi_{i}}+\eta_{i}(t) \frac{E_{i \nu}(t)}{d_{i}}, & \text { if } \beta_{i}(t) \in \Gamma_{\epsilon}^{i}\end{cases}
\end{array}
$$

(where $E_{i \tau}(t)=\left\langle E_{i}(t), \tau\left(\theta_{i}(t)+\phi_{i}\right)\right\rangle / \cos \phi_{i}, \quad E_{i \nu}(t)=\left\langle E_{i}(t)\right.$, $\left.\left.\nu\left(\theta_{i}(t)\right)\right\rangle / \cos \phi_{i}\right)$ assure that $\mathbf{R}_{0}, \mathbf{R}_{1}, \ldots, \mathbf{R}_{n}$ are asymptotically in $(D, \Phi, \mathscr{N})$-formation with leader $\mathbf{R}_{0} \cdot \eta_{i}(t)$ is a bounded continuous function given by

$$
\begin{aligned}
\eta_{i}(t)= & \frac{\left(v_{l_{i}}(t)-W_{l_{i}} / 2\right) \cos \left(\beta_{i}(t)-\phi_{i}\right)}{\left|\left\langle E_{i}(t), \tau\left(\theta_{i}(t)+\phi_{i}\right)\right\rangle\right|} \\
& \wedge \frac{\left(\left(K_{l_{i}}^{+}+\epsilon / 2-\kappa_{l_{i}}(t)\right) \wedge\left(\kappa_{l_{i}}(t)-\left(K_{l_{i}}^{-}-\epsilon / 2\right)\right)\right) d_{i} \cos \phi_{i}}{\left|\left\langle E_{i}(t), \nu\left(\theta_{i}(t)\right)\right\rangle\right|}
\end{aligned}
$$




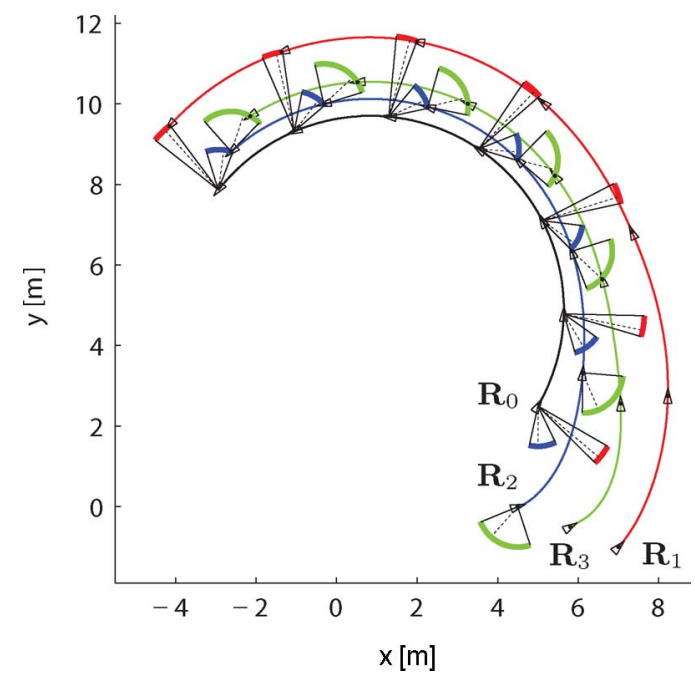

Fig. 8. Trajectory of the robots and arcs of circle $\mathcal{A}_{d_{i}}^{\epsilon}\left(\theta_{l_{i}}(t)+\phi_{i}+\right.$ $\left.\pi,-\arcsin \left(K_{l_{i}}^{+} d_{i} \cos \phi_{i}\right),-\arcsin \left(K_{l_{i}}^{-} d_{i} \cos \phi_{i}\right)\right), i=1,2,3$.

$$
\begin{aligned}
& \wedge \frac{V_{i} \cos \phi_{i}-v_{l_{i}}(t) \cos \left(\beta_{i}(t)-\phi_{i}\right)}{\left|\left\langle E_{i}(t), \tau\left(\theta_{i}(t)+\phi_{i}\right)\right\rangle\right|} \\
& \wedge \frac{v_{l_{i}}(t)\left(K_{i}^{+} d_{i} \cos \left(\beta_{i}(t)-\phi_{i}\right)-\sin \beta_{i}(t)\right)}{\left|\left\langle E_{i}(t), \nu\left(\theta_{i}(t)\right)\right\rangle\right|+\left|K_{i}^{+}\right|\left|\left\langle E_{i}(t), \tau\left(\theta_{i}(t)+\phi_{i}\right)\right\rangle\right|} \\
& \wedge \frac{v_{l_{i}}(t)\left(\sin \beta_{i}(t)-K_{i}^{-} d_{i} \cos \left(\beta_{i}(t)-\phi_{i}\right)\right)}{\left|\left\langle E_{i}(t), \nu\left(\theta_{i}(t)\right)\right\rangle\right|+\left|K_{i}^{-}\right|\left|\left\langle E_{i}(t), \tau\left(\theta_{i}(t)+\phi_{i}\right)\right\rangle\right|} \wedge M_{i}
\end{aligned}
$$

where $M_{i}$ are positive gain constants.

\section{Simulation EXPERIMENTS}

Simulation experiments have been conducted to illustrate the theory and show the effectiveness of the formation controller presented in Section IV.

We considered the $(D, \Phi, \mathscr{N})$-formation reported in Fig. 3, with $d_{1}=2 \mathrm{~m}, d_{2}=d_{3}=1 \mathrm{~m}, \phi_{1}=4 \pi / 9 \quad \mathrm{rad}$ and $\phi_{2}=$ $\phi_{3}=\pi / 6$ rad. The initial conditions of the robots are $\overline{\mathbf{R}}_{0}=$ $(5,2.5, \pi / 3)^{T}, \overline{\mathbf{R}}_{1}=(7,-1, \pi / 4)^{T}, \overline{\mathbf{R}}_{2}=(4.5,0, \pi / 10)^{T}$, and $\overline{\mathbf{R}}_{3}=(5.8,-0.5, \pi / 6)^{T}$. The main leader $\mathbf{R}_{0}$ moves along a circular path, with velocities $v_{0}(t)=1.2 \mathrm{~m} / \mathrm{s}$ and $\omega_{0}(t)=0.25 \mathrm{rad} / \mathrm{s}$. We chose $W_{0}=1 \mathrm{~m} / \mathrm{s}, V_{0}=1.5 \mathrm{~m} / \mathrm{s}, V_{1}=V_{2}=V_{3}=3 \mathrm{~m} / \mathrm{s}, K_{0}^{-}=$ $-0.25 \mathrm{rad} / \mathrm{m}, K_{0}^{+}=0.5 \mathrm{rad} / \mathrm{m}, K_{1}^{-}=K_{2}^{-}=K_{3}^{-}=-0.5 \mathrm{rad} / \mathrm{m}$, and $K_{1}^{-}=K_{2}^{-}=K_{3}^{-}=1 \mathrm{rad} / \mathrm{m}$. With these values, condition (15) is satisfied for $i=1,2,3$, and inequalities (16) hold true. Note that in this case, $\mathscr{L}_{0}=\{1,2\}, \mathscr{L}_{1}=\mathscr{L}_{3}=\emptyset$, and $\mathscr{L}_{2}=\{3\}$. Using the constraints propagation formulas given in Definitions 7 and 8 , we get the bounds $\breve{K}_{0}^{-}, \breve{K}_{0}^{+}$, and $\breve{V}_{0}$. It is easy to verify that $\breve{K}_{0}^{-}, \breve{K}_{0}^{+}$, and $\breve{V}_{0}$ satisfy condition (17). The gains $M_{1}, M_{2}$, and $M_{3}$ in (20) were set to 1. Fig. 8 shows the trajectory of the robots and the arcs of circle $\mathcal{A}_{d_{i}}^{\epsilon}\left(\theta_{l_{i}}(t)+\right.$ $\left.\phi_{i}+\pi,-\arcsin \left(K_{l_{i}}^{+} d_{i} \cos \phi_{i}\right),-\arcsin \left(K_{l_{i}}^{-} d_{i} \cos \phi_{i}\right)\right), i=1,2,3$ are defined in Remark $2\left(\epsilon=10^{-3}\right)$. In order to have a temporal reference in the figure, the vehicles are drawn in every $2 \mathrm{~s}$. Note that after about $9 \mathrm{~s}$, the robots achieve the desired formation. Fig. 9 shows that the norm of the errors $E_{1}, E_{2}$, and $E_{3}$ asymptotically converges to zero.

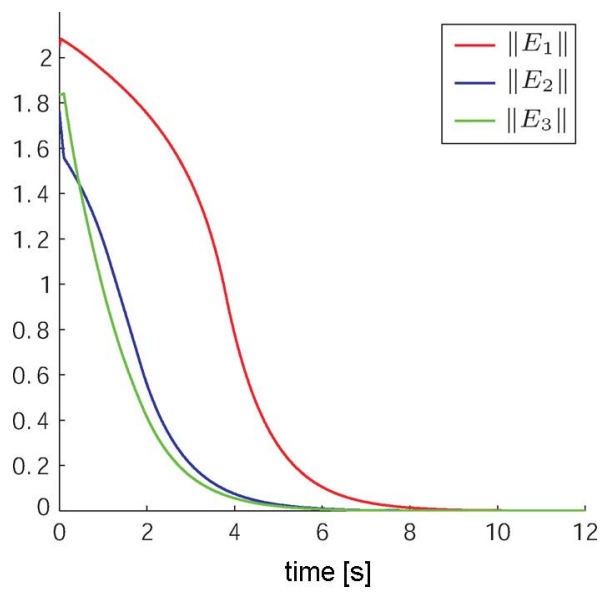

Fig. 9. Norm of the errors $E_{1}, E_{2}$, and $E_{3}$.

\section{CONCLUSION AND FUTURE WORK}

The paper presents a new geometric approach to the stabilization of a hierarchical formation of unicycle robots. The major contribution of the work is the study of the effect of robots' input constraints on the admissible trajectories of the leader guiding the formation. In particular, we provide recursive formulas for the maximum velocity and curvature of the main leader, such that all the other robots can follow the relative leaders while respecting their input constraints. An original formation control strategy is proposed and the asymptotic stabilization is proved.

Future research lines include the extension of our results to vehicles with more involved kinematics (e.g., car-like robots) and the insertion of communication constraints among the agents into our model.

\section{APPENDIX}

\section{A. Proof of Proposition 1}

Let us take any $\epsilon>0$ sufficiently small such that

$$
\left.\begin{array}{l}
\text { if } \left.\phi_{1}<0, \quad-\frac{1}{d_{1} \cos \phi_{1}}\right\}<K_{0}^{-}-\epsilon \\
\text { if } \phi_{1} \geq 0, \quad-\frac{1}{d_{1}},
\end{array}\right\} \begin{aligned}
& \frac{1}{d_{1} \cos \phi_{1}}, \quad \text { if } \phi_{1}>0 \\
& \frac{1}{d_{1}}, \quad \text { if } \phi_{1} \leq 0 \\
& \leq K_{0}^{+}+\epsilon<\breve{K}_{0}^{+} \\
& \breve{K}_{0}^{-}<K_{0}^{-}-\epsilon \leq K_{0}^{+}+\epsilon<\breve{K}_{0} \\
& V_{0} \cos \left(0 \wedge\left(\arcsin \left(\left(K_{0}^{+}+\epsilon\right) d_{1} \cos \phi_{1}\right)-\phi_{1}\right)\right. \\
& \left.\wedge\left(\phi_{1}-\arcsin \left(\left(K_{0}^{-}-\epsilon\right) d_{1} \cos \phi_{1}\right)\right)\right)<V_{1} \cos \phi_{1} .
\end{aligned}
$$

Let the controls be given by (5) and (6), where

$$
\begin{aligned}
\eta_{1}(t)= & \frac{\left(v_{0}(t)-W_{0} / 2\right) \cos \left(\beta_{1}(t)-\phi_{1}\right)}{\left|\left\langle E_{1}(t), \tau\left(\theta_{1}(t)+\phi_{1}\right)\right\rangle\right|} \\
& \wedge \frac{\left(\left(K_{0}^{+}+\epsilon / 2-\kappa_{0}(t)\right) \wedge\left(\kappa_{0}(t)-\left(K_{0}^{-}-\epsilon / 2\right)\right)\right) d_{1} \cos \phi_{1}}{\left|\left\langle E_{1}(t), \nu\left(\theta_{1}(t)\right)\right\rangle\right|} \\
& \wedge \frac{V_{1} \cos \phi_{1}-v_{0}(t) \cos \left(\beta_{1}(t)-\phi_{1}\right)}{\left|\left\langle E_{1}(t), \tau\left(\theta_{1}(t)+\phi_{1}\right)\right\rangle\right|} \\
& \wedge \frac{v_{0}(t)\left(K_{1}^{+} d_{1} \cos \left(\beta_{1}(t)-\phi_{1}\right)-\sin \beta_{1}(t)\right)}{\left|\left\langle E_{1}(t), \nu\left(\theta_{1}(t)\right)\right\rangle\right|+\left|K_{1}^{+}\right|\left|\left\langle E_{1}(t), \tau\left(\theta_{1}(t)+\phi_{1}\right)\right\rangle\right|}
\end{aligned}
$$




$$
\wedge \frac{v_{0}(t)\left(\sin \beta_{1}(t)-K_{1}^{-} d_{1} \cos \left(\beta_{1}(t)-\phi_{1}\right)\right)}{\left|\left\langle E_{1}(t), \nu\left(\theta_{1}(t)\right)\right\rangle\right|+\left|K_{1}^{-}\right|\left|\left\langle E_{1}(t), \tau\left(\theta_{1}(t)+\phi_{1}\right)\right\rangle\right|} \wedge M_{1}
$$

with the convention that $1 / 0=+\infty$ and $M_{1}$ is a positive gain constant. Note that the complexity of $\eta_{1}(t)$ is due to the necessity of satisfying both the curvature and velocity constraints. First of all, we observe that

$$
\exists \bar{t} \geq 0: \beta_{1}(\bar{t}) \in \Gamma_{\epsilon}^{1} .
$$

In fact, suppose, for instance that $K_{1}^{+} \geq 0$; then, if $\forall t \geq 0$, $\beta_{1}(t) \notin \Gamma_{\epsilon}^{1}, \quad$ by $\quad(10), \quad \dot{\beta}_{1}(t)=\omega_{0}(t)-\omega_{1}(t)=\kappa_{0}(t) v_{0}(t)-$ $K_{1}^{+} V_{1} \leq K_{0}^{+} v_{0}(t)-K_{1}^{+} V_{1} \leq\left(\breve{K}_{0}^{+} v_{0}(t)-K_{1}^{+} V_{1}\right)-\epsilon v_{0}(t) \leq$ $\left(\breve{K}_{0}^{+} V_{0}-K_{1}^{+} V_{1}\right)-\epsilon W_{0} \leq-\epsilon W_{0}$, being $\left(\breve{K}_{0}^{+} V_{0}-K_{1}^{+} V_{1}\right) \leq 0$. In fact, suppose for the sake of simplicity that $\cos \left(0 \wedge\left(\arcsin \left(\left(K_{0}^{+}+\right.\right.\right.\right.$ $\left.\left.\left.\epsilon) d_{1} \cos \phi_{1}\right)-\phi_{1}\right) \wedge\left(\phi_{1}-\arcsin \left(\left(K_{0}^{-}-\epsilon\right) d_{1} \cos \phi_{1}\right)\right)\right)=$ $\cos 0=1$, by (12) and (21), we have

$$
\begin{aligned}
\breve{K}_{0}^{+} V_{0}-K_{1}^{+} V_{1} & \leq \frac{V_{1} \cos \phi_{1}}{\sqrt{d_{1}^{2} \cos ^{2} \phi_{1}+\left(\left(K_{1}^{+}\right)^{-1}-d_{1} \sin \phi_{1}\right)^{2}}}-K_{1}^{+} V_{1} \\
& \leq \frac{V_{1}\left(\cos \phi_{1}-\sqrt{\cos ^{2} \phi_{1}+\left(K_{1}^{+} d_{1}-\sin \phi_{1}\right)^{2}}\right)}{\sqrt{d_{1}^{2} \cos ^{2} \phi_{1}+\left(\left(K_{1}^{+}\right)^{-1}-d_{1} \sin \phi_{1}\right)^{2}}} \leq 0 .
\end{aligned}
$$

Therefore, $\beta_{1}(t) \leq-\epsilon W_{0} t+\beta_{1}\left(t_{0}\right) \quad \forall t \geq 0$, which implies straightaway property (23). Set $\beta_{1}^{-1}\left(\Gamma_{\epsilon}^{1}\right)=\left\{t \geq 0 \mid \beta_{1}(t) \in \Gamma_{\epsilon}^{1}\right\}$; then, by (6), $\forall t \in \beta_{1}^{-1}\left(\Gamma_{\epsilon}^{1}\right)$, we have

$$
\begin{aligned}
\dot{\beta}_{1}(t) & =\omega_{0}(t)-\omega_{1}(t) \\
& =\omega_{0}(t)-\frac{v_{0}(t) \sin \beta_{1}(t)}{d_{1} \cos \phi_{1}}+\eta_{1}(t) \frac{E_{1 \nu}(t)}{d_{1}} \\
& =\frac{v_{0}(t)\left(\kappa_{0}(t) d_{1} \cos \phi_{1}-\sin \beta_{1}(t)\right)}{d_{1} \cos \phi_{1}}-\eta_{1}(t) \frac{E_{1 \nu}(t)}{d_{1}}
\end{aligned}
$$

which implies by definition (22) of $\eta_{1}(t)$ that if $\beta_{1}(\bar{t})=\arcsin \left(\left(K_{0}^{+}+\right.\right.$ $\left.\epsilon) d_{1} \cos \phi_{1}\right),\left(\beta_{1}(\bar{t})=\arcsin \left(\left(K_{0}^{-}-\epsilon\right) d_{1} \cos \phi_{1}\right)\right)$, then $\dot{\beta}_{1}(\bar{t}) \leq$ $-\epsilon V_{0} / 2,\left(\dot{\beta_{1}}(\bar{t}) \geq \epsilon W_{0} / 2\right)$. This implies, together with (23), that

$$
\exists \bar{t} \geq 0: \forall t \in[0, \bar{t}), \quad \beta_{1}(t) \notin \Gamma_{\epsilon}^{1} \quad \text { and } \quad \forall t \geq \bar{t}, \quad \beta_{1}(t) \in \Gamma_{\epsilon}^{1}
$$

which gives (14). To prove that constraints (12) are verified for $v_{1}$, note that by (5) and (24), $v_{1}(t)=V_{1} \forall t \in[0, \bar{t})$ and $\forall t \geq \bar{t}$,

$$
v_{1}(t)=\frac{v_{0}(t) \cos \left(\beta_{1}(t)-\phi_{1}\right)}{\cos \phi_{1}}+\eta_{1}(t) E_{1 \tau} .
$$

Therefore, by definition of $\eta_{1}(t)$ and (9), we have

$$
\begin{aligned}
v_{1}(t) & \geq \frac{v_{0}(t) \cos \left(\beta_{1}(t)-\phi_{1}\right)-\eta_{1}(t)\left|\left\langle E_{1}(t), \tau\left(\theta_{1}(t)+\phi_{1}\right)\right\rangle\right|}{\cos \phi_{1}} \\
& \geq \frac{W_{0}}{2} \frac{\cos \left(\beta_{1}(t)-\phi_{1}\right)}{\cos \phi_{1}} .
\end{aligned}
$$

Hence $v_{1}(t)>0 \forall t \geq 0$, by the following property:

$$
\exists c_{1}>0: \cos \left(\beta_{1}(t)-\phi_{1}\right) \geq c_{1} \quad \forall t \geq \bar{t} .
$$

In fact, suppose, for instance, that $\phi_{1}>0$, by (14) and (8), we have $\beta_{1}(t)-\phi_{1} \leq \arcsin \left(\left(K_{0}^{+}+\epsilon\right) d_{1} \cos \phi_{1}\right)-\phi_{1}<\arcsin (1)-$ $\phi_{1}=(\pi / 2)-\phi_{1}$ and $\beta_{1}(t)-\phi_{1} \geq \arcsin \left(\left(K_{0}^{-}-\epsilon\right) d_{1} \cos \phi_{1}\right)-$ $\phi_{1}>-\arcsin \left(\cos \phi_{1}\right)-\phi_{1}=-\pi / 2$, which implies (25) and (13). Furthermore, by (5) and (22), we have $v_{1}(t) \leq V_{1} \forall t \geq 0$. To verify constraints (12) for $\kappa_{1}(t)$, note first of all that they are verified by (5) and (6) if $t \in[0, \bar{t})$. If $t \geq \bar{t}$, since $\eta_{1}(t)>0$ [see (26)], we have $k_{1}(t)$ as shown at the bottom of this page, because, by (22)

$$
\eta_{1}(t) \leq \frac{v_{0}(t)\left(K_{1}^{+} d_{1} \cos \left(\beta_{1}(t)-\phi_{1}\right)-\sin \beta_{1}(t)\right)}{\left|\left\langle E_{1}(t), \nu\left(\theta_{1}(t)\right)\right\rangle\right|+\left|K_{1}^{+}\right|\left|\left\langle E_{1}(t), \tau\left(\theta_{1}(t)+\phi_{1}\right)\right\rangle\right|}
$$

and analogously, $\kappa_{1}(t) \geq K_{1}^{-} \forall t \geq \bar{t}$; therefore, constraints (12) are completely satisfied. To conclude the proof, it remains to verify (11). Differentiating the error $E_{1}(t)$, by (5) and (6), and recalling (4), we get that $\forall t \geq \bar{t}$

$$
\begin{aligned}
\dot{E}_{1}(t)= & v_{0}(t) \tau\left(\theta_{0}(t)\right)-v_{0}(t)\left(\frac{\cos \left(\beta_{1}(t)-\phi_{1}\right)}{\cos \phi_{1}} \tau\left(\theta_{1}(t)\right)\right. \\
& \left.+d_{1} \frac{\sin \beta_{1}(t)}{\cos \phi_{1}} \nu\left(\theta_{1}(t)+\phi_{1}\right)\right)-\eta_{1}(t)\left(E_{1 \tau} \tau\left(\theta_{1}(t)\right)\right. \\
& \left.+E_{1 \nu} \nu\left(\theta_{1}(t)+\phi_{1}\right)\right)=-\eta_{1}(t) E_{1}(t)
\end{aligned}
$$

Therefore, $\left(\frac{d}{d t}\right)\left\|E_{1}(t)\right\|^{2}=-2 \eta_{1}(t)\left\|E_{1}(t)\right\|^{2} \forall t \geq \bar{t}$, and then (11) is satisfied, since the following property holds true:

$$
\exists c>0: \eta_{1}(t) \geq\left(\frac{c}{\left\|E_{1}(t)\right\|} \wedge M_{1}\right) \quad \forall t \geq \bar{t} .
$$

In fact, to verify (26), first of all note that by (9) it follows straightaway that $\left(K_{0}^{+}+\epsilon / 2-\kappa_{0}(t)\right) \wedge\left(\kappa_{0}(t)-\left(K_{0}^{-}-\epsilon / 2\right)\right) \geq$ $\epsilon / 2 \forall t \geq 0$. Moreover, by (21), $V_{1} \cos \phi_{1}-v_{0}(t) \cos \left(\beta_{1}(t)-\phi_{1}\right) \geq$ $V_{1} \cos \phi_{1}-V_{0} \cos \left(0 \wedge\left(\arcsin \left(K_{0}^{+} d_{1} \cos \phi_{1}\right)-\phi_{1}\right) \wedge\left(\phi_{1}-\arcsin \right.\right.$ $\left.\left.\left(K_{0}^{-} d_{1} \cos \phi_{1}\right)\right)\right)=c_{2}>0$, and furthermore, by (14), $\forall t \geq \bar{t}$, we have

$$
\begin{aligned}
& K_{1}^{+}-\frac{\sin \beta_{1}(t)}{d_{1} \cos \left(\beta_{1}(t)-\phi_{1}\right)} \\
& \quad \geq K_{1}^{+}-\frac{\operatorname{sign}\left(K_{0}^{+}+\epsilon\right)}{\sqrt{\left(1 /\left(K_{0}^{+}+\epsilon\right)^{2}\right)-d_{1}^{2} \cos ^{2} \phi_{1}}+d_{1} \sin \phi_{1}}>0
\end{aligned}
$$

since $\left(K_{0}^{+}+\epsilon\right)<\breve{K}_{0}^{+}$, which implies that $\exists c_{3}^{+}>0$, such that $K_{1}^{+} d_{1} \cos \left(\beta_{1}(t)-\phi_{1}\right)-\sin \beta_{1}(t) \geq c_{3}^{+}$; analogously, $\exists c_{3}^{-}>0$, such that $\sin \beta_{1}(t)-K_{1}^{-} d_{1} \cos \left(\beta_{1}(t)-\phi_{1}\right) \geq c_{3}^{-}$. Therefore, bringing together (25) with the previous inequalities, we obtain (26) by definition of $\eta_{1}(t)$.

\section{B. Proof of Theorem 1}

The proof relies on the iterative application of Proposition 1. Let us define the following constants:

$$
\begin{aligned}
& \widehat{K}_{0}^{-}=K_{0}^{-}, \quad \widehat{K}_{0}^{+}=K_{0}^{+} \\
& \widehat{K}_{i}^{-}=\chi_{d_{i}, \phi_{i}}\left(\widehat{K}_{l_{i}}^{-}\right), \quad \widehat{K}_{i}^{+}=\chi_{d_{i}, \phi_{i}}\left(\widehat{K}_{l_{i}}^{+}\right) .
\end{aligned}
$$

$$
\begin{aligned}
\kappa_{1}(t)= & \frac{\omega_{1}(t)}{v_{1}(t)}=\frac{v_{0}(t) \sin \beta_{1}(t)+\eta_{1}(t)\left\langle E_{1}(t), \nu\left(\theta_{1}(t)\right)\right\rangle}{d_{1} v_{0}(t) \cos \left(\beta_{1}(t)-\phi_{1}\right)+\eta_{1}(t)\left\langle E_{1}(t), \tau\left(\theta_{1}(t)+\phi_{1}\right)\right\rangle} \\
& \leq \frac{v_{0}(t) \sin \beta_{1}(t)+\eta_{1}(t)\left|\left\langle E_{1}(t), \nu\left(\theta_{1}(t)\right)\right\rangle\right|}{d_{1} v_{0}(t) \cos \left(\beta_{1}(t)-\phi_{1}\right)-\eta_{1}(t)\left(\operatorname{sign} K_{1}^{+}\right)\left|\left\langle E_{1}(t), \tau\left(\theta_{1}(t)+\phi_{1}\right)\right\rangle\right|} \leq K_{1}^{+}
\end{aligned}
$$


We want to verify that $\forall i=1, \ldots, n$, we have

$$
\breve{K}_{i}^{-}<\widehat{K}_{i}^{-}, \quad \widehat{K}_{i}^{+}<\breve{K}_{i}^{+} .
$$

Note that the interval $\left[\widehat{K}_{i}^{-}, \widehat{K}_{i}^{+}\right]$represents the curvature range necessary for the ith robot to follow its relative leader. On the other hand, the interval $\left[\breve{K}_{i}^{-}, \breve{K}_{i}^{+}\right]$represents the curvature range that is compatible with the curvature constraints of the robots contained in the subtree having the robot ith as root. We will prove only the second inequality in (27), since the first one follows analogously. Set $i=1$, by hypothesis (17) and monotonicity of $\chi_{d_{1}, \phi_{1}}$, we have

$$
\begin{aligned}
\widehat{K}_{1}^{+}= & \chi_{d_{1}, \phi_{1}}\left(\widehat{K}_{0}^{+}\right)=\chi_{d_{1}, \phi_{1}}\left(K_{0}^{+}\right) \\
& <\chi_{d_{1}, \phi_{1}}\left(\breve{K}_{0}\right) \leq \chi_{d_{1}, \phi_{1}}\left(\chi_{d_{1}, \phi_{1}}^{-1}\left(\breve{K}_{1}^{+}\right)\right)=\breve{K}_{1}^{+} .
\end{aligned}
$$

By induction, let suppose that the second in (27) holds for $1 \leq i \leq$ $m-1$ (with $1<m \leq n$ ). Since the property holds for $i=l_{m}$, being $1 \leq l_{m} \leq m-1$, we have, as before,

$$
\begin{aligned}
\widehat{K}_{m}^{+}= & \chi_{d_{m}, \phi_{m}}\left(\widehat{K}_{l_{m}}^{+}\right)<\chi_{d_{m}, \phi_{m}}\left(\breve{K}_{l_{m}}\right) \\
& \leq \chi_{d_{m}, \phi_{m}}\left(\chi_{d_{m}, \phi_{m}}^{-1}\left(\breve{K}_{m}^{+}\right)\right)=\breve{K}_{m}^{+} .
\end{aligned}
$$

Therefore, the second in (27) holds for $i=m$. For any $\rho>0$, let us define

$$
\widehat{K}_{0, \rho}^{-}=K_{0}^{-}, \quad \widehat{K}_{0, \rho}^{+}=K_{0}^{+}
$$

and $\forall i=1, \ldots, n$

$$
\widehat{K}_{i, \rho}^{-}=\chi_{d_{i}, \phi_{i}}\left(\widehat{K}_{l_{i}, \rho}^{-}-\rho\right), \quad \widehat{K}_{i, \rho}^{+}=\chi_{d_{i}, \phi_{i}}\left(\widehat{K}_{l_{i}, \rho}^{+}+\rho\right) .
$$

By the continuity of $\chi_{d_{i}, \phi_{i}}$, we get that $\lim _{\rho \rightarrow 0} \widehat{K}_{i, \rho}^{ \pm}=$ $\widehat{K}_{i}^{ \pm} \forall i=1, \ldots, n$. Therefore, by (27), there exists a $\rho>0$, such that $\forall i=1, \ldots, n$, we have

$$
\breve{K}_{i}^{-}<\widehat{K}_{i, \rho}^{-} \leq \widehat{K}_{i, \rho}^{+}<\breve{K}_{i}^{+} .
$$

Let $\epsilon>0$ be sufficiently small, such that

$$
\breve{K}_{i}^{-}<\widehat{K}_{i, \rho}^{-}-\epsilon<\widehat{K}_{i, \rho}^{-} \leq \widehat{K}_{i, \rho}^{+}<\widehat{K}_{i, \rho}^{+}+\epsilon<\breve{K}_{i}^{+}
$$

and let us set $\forall i=1, \ldots, n$,

$$
\begin{aligned}
W_{i}= & \frac{W_{l_{i}}}{2 \cos \phi_{i}} \cos \left(\left(\arcsin \left(\left(\widehat{K}_{l_{i}, \rho}^{+}+\epsilon\right) d_{i} \cos \phi_{i}\right)-\phi_{i}\right)\right. \\
& \left.\vee\left(\phi_{i}-\arcsin \left(\left(\widehat{K}_{l_{i}, \rho}^{-}-\epsilon\right) d_{i} \cos \phi_{i}\right)\right)\right) .
\end{aligned}
$$

We now prove (18) and (19) by induction. First of all, let us take $i=1$; in this case, (15) is exactly (8). If we apply Proposition 1 with $K_{1}^{ \pm}=\widehat{K}_{1, \rho}^{ \pm}$, noting that $\breve{K}_{0}^{ \pm}$in Proposition 1 is just $\chi_{d_{1}, \phi_{1}}^{-1}\left(K_{1}^{ \pm}\right)$, we get

$$
\breve{K}_{0}^{ \pm}=\chi_{d_{1}, \phi_{1}}^{-1}\left(\widehat{K}_{1, \rho}^{ \pm}\right)=\chi_{d_{1}, \phi_{1}}^{-1}\left(\chi_{d_{1}, \phi_{1}}\left(\widehat{K}_{0, \rho}^{ \pm} \pm \rho\right)\right)=K_{0}^{ \pm} \pm \rho
$$

which implies condition (10). Therefore, by Proposition 1, we have

$$
\lim _{t \rightarrow+\infty} E_{1}(t)=0
$$

and

$$
\begin{aligned}
& 0<W_{1} \leq v_{1}(t) \leq V_{1} \\
& K_{1}^{-} \leq \breve{K}_{1}^{-}<\widehat{K}_{1, \rho}^{-} \leq \kappa_{1}(t) \leq \widehat{K}_{1, \rho}^{+}<\breve{K}_{1}^{+} \leq K_{1}^{+} .
\end{aligned}
$$

This means that (18) is verified for $i=1$. Let us suppose by induction that (18) holds for $1 \leq i \leq m-1$ (with $1<m \leq n$ ), and let us take, in Proposition $1, K_{0}^{ \pm}=\widehat{K}_{l_{m}, \rho}^{ \pm}$and $K_{1}^{ \pm}=\widehat{K}_{m, \rho}^{ \pm}$. Therefore, $\widehat{K}_{0}^{ \pm}$in Proposition 1 is given by

$$
\begin{aligned}
\widehat{K}_{0}^{ \pm} & =\chi_{d_{m}, \phi_{m}}^{-1}\left(\widehat{K}_{m, \rho}^{ \pm}\right) \\
& =\chi_{d_{m}, \phi_{m}}^{-1}\left(\chi_{d_{m}, \phi_{m}}\left(\widehat{K}_{l_{m}, \rho}^{ \pm} \pm \rho\right)\right)=\widehat{K}_{l_{m}, \rho}^{ \pm} \pm \rho .
\end{aligned}
$$

Moreover, by (15), (28), and the inductive hypotheses, we have

$$
\left.\begin{array}{l}
\text { if } \left.\phi_{m}<0, \quad-\frac{1}{d_{m} \cos \phi_{m}}\right\} \leq \\
\text { if } \phi_{m} \geq 0, \quad-\frac{1}{d_{m}} \quad
\end{array}\right\} \begin{aligned}
& K_{l_{m}}^{-} \leq \breve{K}_{l_{m}}^{-}<\widehat{K}_{l_{m}, \rho}^{-} \leq \kappa_{l_{m}}(t) \leq \widehat{K}_{l_{m}, \rho}^{+}<\breve{K}_{l_{m}}^{+} \leq K_{l_{m}}^{+} \\
& \leq \begin{cases}\frac{1}{d_{m} \cos \phi_{m}}, & \text { if } \phi_{m}>0 \\
\frac{1}{d_{m}}, & \text { if } \phi_{m} \leq 0 .\end{cases}
\end{aligned}
$$

Therefore, all the hypotheses of Proposition 1 are verified if we take, in Proposition $1, K_{0}^{ \pm}=\widehat{K}_{l_{m}, \rho}^{ \pm}, K_{1}^{ \pm}=\widehat{K}_{m, \rho}^{ \pm}$, and $\breve{K}_{0}^{ \pm}=$ $\widehat{K}_{l_{m}, \rho}^{ \pm} \pm \rho$. Then, applying it again, we obtain that (18) holds for $i=m$ and $\lim _{t \rightarrow+\infty} E_{m}(t)=0$. Finally, (19) is a straight consequence of Remark 2 applied to each leader-follower unit $\left(\mathbf{R}_{l_{i}}, \mathbf{R}_{i}\right)$.

\section{REFERENCES}

[1] F. Bullo, J. Cortés, and S. Martínez, Distributed Control of Robotic Networks (Applied Mathematics Series). Princeton, NJ: Princeton Univ. Press, 2009.

[2] L. E. Parker, "Multiple mobile Robot systems," in Handbook of Robotics, B. Siciliano and O. Khatib, Eds., New York: Springer-Verlag, 2008, ch. 40, pp. 921-941.

[3] V. Kumar, D. Rus, and G. S. Sukhatme, "Networked robots," in Handbook of Robotics, B. Siciliano and O. Khatib, Eds. New York: SpringerVerlag, 2008, ch. 41, pp. 943-958.

[4] A. Jadbabaie, J. Lin, and A. S. Morse, "Coordination of groups of mobile autonomous agents using nearest neighbor rules," IEEE Trans. Autom. Control, vol. 48, no. 6, pp. 988-1001, Jun. 2003.

[5] R. Olfati-Saber, J. A. Fax, and R. M. Murray, "Consensus and cooperation in networked multi-agent systems," Proc. IEEE, vol. 95, no. 1, pp. 215233, Jan. 2007

[6] H. Ando, Y. Oasa, I. Suzuki, and M. Yamashita, "Distributed memoryless point convergence algorithm for mobile robots with limited visibility," IEEE Trans. Robot. Autom., vol. 15, no. 5, pp. 818-828, Oct. 1999.

[7] J. Lin, A. S. Morse, and B. D. O. Anderson, "The multi-agent rendezvous problem. Part 1: The synchronous case," SIAM J. Control Optim., vol. 46, no. 6, pp. 2096-2119, 2007.

[8] J. Cortés, S. Martínez, T. Karatas, and F. Bullo, "Coverage control for mobile sensing networks," IEEE Trans. Robot. Autom., vol. 20, no. 2, pp. 243-255, Apr. 2004

[9] J. Meng and M. Egerstedt, "Distributed coordination control of multiagent systems while preserving connectedness," IEEE Trans. Robot., vol. 23, no. 4, pp. 693-703, Aug. 2007.

[10] D. V. Dimarogonas and K. J. Kyriakopoulos, "Connectedness preserving distributed swarm aggregation for multiple kinematic robots," IEEE Trans. Robot., vol. 24, no. 5, pp. 1213-1223, Oct. 2008.

[11] K. H. Tan and M. A. Lewis, "High precision formation control of mobile robots using virtual structures," Auton. Robots, vol. 4, no. 4, pp. 387-403, 1997.

[12] T. Balch and R. C. Arkin, "Behavior-based formation control for multirobot teams," IEEE Trans. Robot. Autom., vol. 14, no. 6, pp. 926-939, Dec. 1998.

[13] M. Egerstedt and X. Hu, "Formation constrained multi-agent control," IEEE Trans. Autom. Control, vol. 17, no. 6, pp. 947-951, Dec. 2001.

[14] P. Tabuada, G. J. Pappas, and P. Lima, "Motion feasibility of multi-agent formations," IEEE Trans. Robot., vol. 21, no. 3, pp. 387-392, Jun. 2005

[15] W. Dong and J. A. Farrell, "Cooperative control of multiple nonholonomic mobile agents," IEEE Trans. Autom. Control, vol. 53, no. 6, pp. 1434 1448, Jul. 2008. 
[16] J. A. Fax and R. M. Murray, "Information flow and cooperative control of vehicle formations," IEEE Trans. Autom. Control, vol. 49, no. 9, pp. 1465-1476, Sep. 2004.

[17] Z. Lin, B. A. Francis, and M. Maggiore, "Necessary and sufficient graphical conditions for formation control of unicycles," IEEE Trans. Autom. Control, vol. 50, no. 1, pp. 121-127, Jan. 2005.

[18] T. I. Fossen, Guidance and Control of Ocean Vehicles. New York: Wiley, 1994.

[19] D. B. Edwards, T. A. Bean, D. L. Odell, and M. J. Anderson, "A leaderfollower algorithm for multiple AUV formations," in Proc. IEEE/OES Auton. Underwater Vehicles, 2004, pp. 40-46.

[20] T. J. Koo and S. M. Shahruz, "Formation of a group of unmanned aerial vehicles (UAVs)," in Proc. Amer. Control Conf., 2001, pp. 69-74.

[21] L. E. Buzogany, M. Pachter, and J. J. D'Azzo, "Automated control of aircraft in formation flight," in Proc. AIAA Guid., Navigat., Control Conf., 1993, pp. 1349-1370.

[22] H. Schaub, S. R. Vadali, J. L. Junkins, and K. T. Alfriend, "Spacecraft formation flying control using mean orbit elements," J. Astronaut. Sci., vol. 48, no. 1, pp. 69-87, 2000.

[23] R. Burns, C. A. McLaughlin, J. Leitner, and M. Martin, "TechSat21: Formation design, control and simulation," in Proc. IEEE Aerosp. Conf., 2000, vol. 7, pp. 19-25.

[24] A. K. Das, R. Fierro, V. Kumar, J. P. Ostrowsky, J. Spletzer, and C. Taylor, "A vision-based formation control framework," IEEE Trans. Robot. Autom., vol. 18, no. 5, pp. 813-825, Oct. 2002.

[25] H. G. Tanner, G. J. Pappas, and V. Kumar, "Leader-to-formation stability," IEEE Trans. Robot. Autom., vol. 20, no. 3, pp. 443-455, Jun. 2004

[26] F. Morbidi, G. L. Mariottini, and D. Prattichizzo, "Vision-based range estimation via Immersion and Invariance for robot formation control," in Proc. IEEE Int. Conf. Robot. Autom., 2008, pp. 504-509.

[27] L. Consolini, F. Morbidi, D. Prattichizzo, and M. Tosques, "Leaderfollower formation control of nonholonomic mobile robots with input constraints," Automatica, vol. 44, no. 5, pp. 1343-1349, 2008.

\section{Shortest Paths to Obstacles for a Polygonal Dubins Car}

Paolo Robuffo Giordano and Marilena Vendittelli

\begin{abstract}
In this paper, we characterize the time-optimal trajectories leading a Dubins car in collision with the obstacles in its workspace. Due to the constant velocity constraint characterizing the Dubins car model, these trajectories form a sufficient set of shortest paths between any robot configuration and the obstacles in the environment. Based on these paths, we define and give the algorithm for computing a distance function that takes into account the nonholonomic constraints and captures the nonsymmetric nature of the system. The developments presented here assume that the obstacles and the robot are polygons although the methodology can be applied to different shapes.
\end{abstract}

Index Terms-Dubins car, Pontryagin's maximum principle, shortest paths.

\section{INTRODUCTION}

In his pioneering work [1], Dubins determined the continuously differentiable curves of minimal length between any two points in the plane with assigned initial and final tangent and subject to curvature

Manuscript received August 30, 2008; revised December 4, 2008. First published February 2, 2009; current version published October 9, 2009. This paper was recommended for publication by Associate Editor E. Papadopoulos and Editor J.-P. Laumond upon evaluation of the reviewers' comments.

The authors are with the Dipartimento di Informatica e Sistemistica, Università di Roma "La Sapienza", 00185 Rome, Italy (e-mail: robuffo@ dis.uniroma1.it; venditt@ dis.uniroma1.it).

Color versions of one or more of the figures in this paper are available online at http://ieeexplore.ieee.org.

Digital Object Identifier 10.1109/TRO.2008.2011421 constraints. These curves are time-optimal trajectories for a wheeled mobile robot with unitary speed and bounded steering velocity. For this reason, a vehicle with constant driving velocity is known in the literature as the Dubins car. The interest arisen by this system is due to both the theoretical challenges that it provides and its practical relevance in modeling the kinematics of road vehicles, aircrafts cruising at constant altitude, or sea vessels.

The difficulty of planning for a wheeled mobile robot derives from the pure rolling constraint of the wheels that prevents the vehicle to move instantaneously toward certain directions. As a consequence, not all the paths in the configuration space are feasible for this robot, and the Euclidean metric is not appropriate for determining the distance to obstacles populating the robot environment. In a previous work [2], we have defined and showed how to compute a distance between a pointwise robot and the obstacles in its environment by taking into account the nonholonomic constraints acting on the vehicle. In this paper, we consider both the nonholonomic constraint and the shape of the robot. Specifically, we define and compute a distance in the robot configuration space that takes into account the nonholonomic constraints of the vehicle. The computation does not require the explicit representation of the configuration space obstacles and is applicable to robots and obstacles that can be modeled as planar polygons. This distance function is particularly relevant in the context of motion planners relying on obstacle distance computation, like skeletonization, potential field methods, and even sampling-based motion planners. See, e.g., [3] for an interesting discussion on the use of the obstacle distance information in planning algorithms. In particular, the correct metric information provided by the distance developed in this paper can improve the efficiency of sampling-based methods: by computing the distance to the obstacles in the environment, it is possible to adjust the resolution at which configurations along local paths are checked for collision, in the line of [4]. This avoids the difficult resolution tuning step and provides an exact solution to the collision check test for local paths.

Our work relies on Dubins' results but adopts the optimal control approach proposed in [5] and [6]. In particular, the study of transversality conditions allows selecting a sufficient family of time-optimal trajectories whose length will determine the distance to the obstacles. A preliminary version of this paper has been presented in [7]. Here, we present a revised work extended with detailed proofs and formal equations for distance computation. The paper is organized as follows. In Section II, we summarize the basic results on time-optimal trajectories for the Dubins car. Due to the constant velocity constraint, these trajectories also minimize the path length. This is exactly the property that we exploit in defining the distance function in Section III where we also give the necessary conditions satisfied by the shortest paths bringing the robot in collision with obstacles. The distance function is based on the length of the shortest paths belonging to a sufficient family. In Section IV, we show how to reduce the cardinality of this set of paths to lower the distance computation complexity, and in Section V, we provide the formal system of equations needed for implementation. These are used in Section VI to obtain the isodistance curves on the $x y$ plane for two robots with different shapes.

\section{BACKGROUND MATERIAL}

The kinematic model of the Dubins car considered in this paper is

$$
\dot{\xi}(t)=f(\xi(t), u(t))=g_{1}(\xi(t))+g_{2}(\xi(t)) u(t)
$$

where $\xi \in R^{2} \times \mathcal{S}^{1}$ denotes the configuration of the vehicle, given by the position $\left(x_{r}, y_{r}\right)$ of a reference point $P$, chosen as the mid point of the rear wheels' axle, and by the orientation $\theta$ of the vehicle w.r.t. the abscissa axis of a fixed reference frame. The drift and the input 